\title{
Marie Goyon
}

Chercheure associée, Centre de Recherches et d'études anthropologiques (CREA)

Université Lumière, Lyon 2

(2007)

\section{“L'Indianité en tant qu'ethnogénèse: exemple de mobilisation dans l'art contemporain amérindien”}

\author{
Un document produit en version numérique par Jean-Marie Tremblay, bénévole, \\ professeur de sociologie au Cégep de Chicoutimi \\ Courriel: jean-marie_tremblay@uqac.ca \\ Site web pédagogique : http://www.uqac.ca/jmt-sociologue/ \\ Dans le cadre de: "Les classiques des sciences sociales" \\ Une bibliothèque numérique fondée et dirigée par Jean-Marie Tremblay, \\ professeur de sociologie au Cégep de Chicoutimi \\ Site web: http://classiques.uqac.ca/ \\ Une collection développée en collaboration avec la Bibliothèque \\ Paul-Émile-Boulet de l'Université du Québec à Chicoutimi \\ Site web: http://bibliotheque.uqac.ca/
}




\section{Politique d'utilisation de la bibliothèque des Classiques}

Toute reproduction et rediffusion de nos fichiers est interdite, même avec la mention de leur provenance, sans l'autorisation formelle, écrite, du fondateur des Classiques des sciences sociales, Jean-Marie Tremblay, sociologue.

Les fichiers des Classiques des sciences sociales ne peuvent sans autorisation formelle:

- être hébergés (en fichier ou page web, en totalité ou en partie) sur un serveur autre que celui des Classiques.

- servir de base de travail à un autre fichier modifié ensuite par tout autre moyen (couleur, police, mise en page, extraits, support, etc...),

Les fichiers (.html, .doc, .pdf., .rtf, .jpg, .gif) disponibles sur le site Les Classiques des sciences sociales sont la propriété des Classiques des sciences sociales, un organisme à but non lucratif composé exclusivement de bénévoles.

Ils sont disponibles pour une utilisation intellectuelle et personnelle et, en aucun cas, commerciale. Toute utilisation à des fins commerciales des fichiers sur ce site est strictement interdite et toute rediffusion est également strictement interdite.

L'accès à notre travail est libre et gratuit à tous les utilisateurs. C'est notre mission.

Jean-Marie Tremblay, sociologue

Fondateur et Président-directeur général, LES CLASSIQUES DES SCIENCES SOCIALES. 
Cette édition électronique a été réalisée par Jean-Marie Tremblay, bénévole, professeur de sociologie au Cégep de Chicoutimi à partir de :

\section{Marie Goyon}

“L'Indianité en tant qu'ethnogénèse : exemple de mobilisation dans l'art contemporain amérindien”.

Un article publié dans la revue Parcours anthropologiques, no 6, 2007, pp. 92-112.

["Pourrait-on considérer la notion d'« indianité » comme une ethnogénèse, notamment dans le cadre de sa mobilisation en tant que catégorie identificatoire, dans le monde de l'art contemporain ?’]

[Autorisation formelle accordée par l'auteure le 14 avril 2008 de diffuser ce texte dans Les Classiques des sciences sociales.]

Courriel : marie.goyon@univ-lyon2.fr

Site web : http://mariegoyon.free.fr/

Polices de caractères utilisée :

Pour le texte: Times New Roman, 14 points.

Pour les citations : Times New Roman, 12 points.

Pour les notes de bas de page : Times New Roman, 12 points.

Édition électronique réalisée avec le traitement de textes Microsoft Word 2004 pour Macintosh.

Mise en page sur papier format : LETTRE (US letter), 8.5'’ $\mathrm{x}$ 11'”)

Édition numérique réalisée le 27 avril 2008 à Chicoutimi, Ville de Saguenay, province de Québec, Canada. 


\section{Table des matières}

$\underline{\text { Introduction }}$

Qu'est-ce que l'Indianité ?

Indianité juridique

Indianité politique

Indianité entre symboles et stéréotypes

L'Indianité comme dépassement des catégories de l'art tribal vers l'art « universel »

Le paradoxe des identités

Art et dépassement des catégories

$\underline{\text { En conclusion }}$

Bibliographie 


\section{Marie Goyon \\ Chercheure associée, Centre de Recherches et d'études anthropologiques (CREA) \\ Université Lumière, Lyon 2 \\ "L'Indianité en tant qu'ethnogénèse : exemple de mobilisation dans l'art contemporain amérindien”.}

Un article publié dans la revue Parcours anthropologiques, no 6, 2007, pp. 92-112.

\section{Introduction}

Face à des concepts anthropologiques qui parfois se perdent, s'épuisent ou se connotent, il est toujours intéressant de s'arrêter un instant et de les questionner. Cet exercice est bien évidemment nécessaire à toute démarche scientifique, et même salutaire lorsque le contexte économique et social révèle des tensions et conflits particulièrement accentués : les tensions et inventions sont bien sûr toujours présentes et apparaissent comme les moteurs d'une société, mais elles échappent parfois à ceux qui tentent de les penser et prennent des proportions qui les dépassent. Il semble que ce soit le cas aujourd'hui face à ce que l'on peine à désigner sous les termes de "globalisation", "mondialisation”, "village-monde”. Exercer alors une certaine réflexivité sur les productions et les épistémologies de sa discipline, en l'occurrence l'anthropologie, permet d'avancer d'un oeil renouvelé. Dans cette perspective, il sera ici question d'un concept choisi parmi ceux qui ont pu être rejetés, parfois de façon très rapide et même péremptoire, la connotation péjorative étant allée plus vite que l'exercice de la critique : je pense justement au procès dont la notion que nous 
allons discuter ici, l'ethnogénèse, a été victime dans les années $1970{ }^{1}$. Il est dommage de rejeter un terme sémantiquement intéressant à cause des usages très divers et toujours contextuels qui en ont été faits, cela revenant, pour utiliser une expression très imagée, "à jeter le bébé avec l'eau du bain”...

En effet, dans un contexte contemporain de mobilité et de processus, certains concepts peuvent trouver une nouvelle jeunesse. C'est le cas de la notion d'ethnogénèse, telle qu'on la reprendra ici, à sa base étymologique, en tant que "processus de formation d'un peuple, d'une identité culturelle" et sous la dimension plutôt dynamique et militante qui en est proposée par l'anthropologie culturelle américaine. L’etnnogénèse pourra alors renvoyer à des phénomènes et discours observés dans la pratique du terrain (pour moi le terrain amérindien et canadien), au contact d'acteurs qui tentent de se redéfinir et de se réunir autour de valeurs et symboles, en vue de la construction d'une appartenance particulière, relevant d'une conception plurielle et mouvante de l’identité.

La question plus spécifique que je propose d'aborder dans cet article est la suivante : pourrait-on considérer la notion d' «indianité » comme une ethnogénèse, notamment dans le cadre de sa mobilisation en tant que catégorie identificatoire, dans le monde de l'art contemporain?

1 Pour en savoir plus, on pourra consulter le résumé de l'historique de la notion, proposé dans Bonte P. et Izard M. (ed), Dictionnaire de l'ethnologie et de l'anthropologie, PUF, Paris, 2000, pp. 787-89. On pourra ainsi constater les multiples usages et interprétations du terme selon les époques et les traditions anthropologiques nationales, de la France à la Russie soviétique, en passant par les Etats-Unis. C'est cette dernière acception, née de l'anthropologie culturelle nord-américaine, qui sera plus particulièrement pertinente face à la conception évoquée ici, de l'Indianité dans l'art. L'ethnogénèse ainsi envisagée renvoie à des processus de reviviscence ou d'émergence d'une conscience collective de groupes souvent minoritaires, dont l'élément clé est le besoin de démarquage vis-à-vis du groupe dominant. 
Afin de proposer des modalités de réponses à cette interrogation, j'avancerai dans un premier temps quelques définitions possibles de l'Indianité 2 , sous ses aspects juridique, politique et symbolique. Dans un deuxième temps, j'aborderai des exemples de la mobilisation de cette Indianité dans l'art amérindien (États-Unis et Canada) depuis les années 1960. Ces exemples amèneront à critiquer la notion d' « authenticité » de l'oeuvre et du primitivisme dans les contextes muséographiques. L'Indianité sera interrogée ensuite comme une possibilité de dépassement des catégories de l'art tribal vers l'art « universel », et notamment comme instrument de visibilité des artistes d'origine autochtone sur la scène internationale. Enfin, le concept d'ethnoscape ${ }^{3}$ sera envisagé comme une perspective d'analyse dynamique de cette création des imaginaires négociés, entre art occidental et non-occidental, à l'aune d'un changement d'échelle radical.

\section{Qu'est-ce que l’Indianité ?}

$\underline{\text { Retour à la table des matières }}$

Le terme d'indianité n'est pas simple à manier, car il revêt diverses significations selon les contextes de son utilisation. En anglais, il existe même deux termes qui renvoient en français à une même traduction : indianness et indianity, indianité. La différence entre les deux est subtile à définir et la plupart des dictionnaires n’y parviennent d'ailleurs pas. Cependant, on peut principalement s'attacher au fait que le suffixe "ness" renvoit à une essence, une immanence, une qualité de substance profonde et symbolique dont l'être ne peut se départir (on pourrait traduire cette idée en français par la notion substanti-

2 J'utiliserai la forme à majuscule Indianité quand il s'agira de la notion telle qu'elle est mobilisée par les acteurs de ce terrain, des artistes contemporains d'origine autochtone se réclamant de l'Indianité.

3 Appaduraï Arjun, Après le colonialisme. Les conséquences culturelles de la globalisation, Payot, Paris, 2001. 
viste de "nature" des choses, des êtres), qui induit donc une certaine fatalité, un déterminisme ; alors que le suffixe "ity” s'appuie sur une dimension dynamique de construction historique de l'identité. Il s'agit également d'une caractéristique identitaire de l'être, peut-être pour part inconsciente et innée (la naissance, le sang), mais qui peut aussi être choisie, puis élaborée.

J'emploierai donc ici le mot “indianité” tel qu'il est mobilisé en Amérique du Nord, sous la terminologie indianity, et je m'attacherai à questionner la dimension de ce référent dans le contexte autochtone et artistique. Nous verrons que cette notion renvoie au départ à une distinction identitaire (vis-à-vis des «autres», les vastes «nonindiens »), ne relevant plus ni de la «tribu », ni de la 3 «nation », et relaye bien la référence à une identité commune transtribale et transnationale (Canada et Etats-Unis), née avec le panindianisme politique des années 1960 (sujet développé en second point, "Indianité politique”). Il s’agira donc de savoir si l'on peut considérer que l'Indianité tend à former une nouvelle identité culturelle, fondée sur des communs de langue, culte, "philosophie », repères esthétiques, transtribaux et transnationaux.

Dans l'hypothèse positive, alors dans quel(s) processus s'inscritelle, quelles sont les stratégies à l'oeuvre dans ce mouvement de l'Indianité ? En d'autres termes, comment et pourquoi préfère-t-on se définir (ou "se vendre ») comme " artiste indien », comme " art indien », ou au contraire pourquoi récuse-t-on cette étiquette ?

Pour bien comprendre la notion d'Indianité, il faut donc en premier lieu la considérer sous trois aspects, que nous appellerons juridique, politique et symbolique. 


\section{Indianité juridique}

$\underline{\text { Retour à la table des matières }}$

D’un point de vue juridique, l'indianité existe de façon très évasive au sein de la Loi sur les Indiens (Indian Act), régissant depuis 1876, au Canada, le statut des Amérindiens. Les textes font ainsi état de la " quiddité indienne », comme statuts et valeurs " fondamentales » de l'”indianité". C'est indianness, et non indianity, qui est le terme utilisé dans ces textes, ce qui n'a rien d'anodin si l'on considère les définitions que nous avons proposé plus haut des deux termes. Le terme d'indianity n'apparaît pas, car trop marqué politiquement: l'utiliser équivaudrait à reconnaître un pouvoir aux populations autochtones, celui de se déterminer, au propre comme au figuré.

En effet, il est par exemple dit dans la loi constitutionnelle de 1867, préexistant à la Loi sur les Indiens, que les gouvernements provinciaux du Canada ne peuvent en aucun cas affecter "les Indiens dans leur indianité” ${ }^{4}$. Pour nous éclairer sur la signification donnée à ce terme par le gouvernement canadien, il faut savoir qu'il a par ailleurs été statué que l'essentiel de l'indianité ne comprenait pas les relations de travail, ni la conduite de véhicules à moteur... Mais là encore, toujours pas d'indications claires fournies sur ce qu'est cette "indianité”, et ce qu’elle implique.

Ce qui apparaît, c'est que la notion renvoie à une conception substantialiste de l'identité, suffisamment floue et romantique, pour ne pas avoir à assumer de conséquences pratiques, c'est-à-dire économiques, politiques et sociales. L'Indien est avant tout “autre”, et l'on ne ga-

4 Pour prendre connaissance des statuts successifs de l'Indien, voir par exemple chez Dupuis Renée, La question indienne au Canada, Boréal, Montréal, 1991. 
gnerait pas, pour maintenir sa domination, à le définir de manière trop précise, car cela risquerait de lui donner des droits...

La notion est ainsi toujours plus esquissée que définie. Et lorsqu'elle l'est, c'est souvent par défaut. C'est-à-dire par exemple que le progrès (les véhicules à moteur que nous évoquions plus haut) et "l'utilité sociale", la productivité (les relations de travail) ne sont pas des caractéristiques indiennes. De là à dire que l'archaïsme et l'oisiveté sont des traits spécifiques du "bon sauvage” que demeure l'Indien aux yeux de la loi, il n'y a qu'un pas... Qui sera franchi avec l'exemple suivant.

En fait, le seul exemple concret d'indianité a été donné dans l'arrêt Dick (1985), concernant la chasse hors saison d'un cerf par un Indien nommé Dick, contrevenant ainsi à la Wildlife Act (1979). S'exprimant au nom de la Cour, le juge Beetz a tenu pour acquis, sans toutefois se prononcer sur la question, qu'une loi provinciale en matière de chasse ne s'appliquait pas d'elle-même aux membres d'une bande indienne lorsqu'ils chassent, parce que ces activités étaient « au coeur même de leur existence et de leur être » (Arrêt Dick c. La Reine, [1985], 2. R.C.S 309). La nature du sauvage indien est donc de chasser, et il ne peut s'en empêcher puisqu'il s'agit d'une caractéristique de son être... L’Indianité aux yeux de la loi apparaît comme une propriété intrinsèque de l'Indien, une sorte d'essence que le terme indianness souligne.

Mais qu'est-ce alors qu'être Indien, quand l’indianité est indéfinissable ou marquée par l'ethnocentrisme colonial, et quand les statuts juridiques sont extrêmement complexes et multiples ${ }^{5}$, quand la réalité

5 L'Indian Act distingue les « Indiens des traités » correspondant aux autochtones ayant été répertoriés entre 1850 et 1923 lors de la signature des traités, ainsi que leurs descendants. Ils bénéficient de tous les avantages des traités (sur les réserves, gratuité de l'éducation, aide sociale, programmes de logement, défiscalisation...) et ont des droits juridiques. Les autres sont les « Indiens inscrits », qui ne sont pas assujettis aux traités mais bénéficient des avantages liés à ces derniers. Leurs ancêtres sont ceux qui n’ont pas signé, 
sociale et historique est celle de l'assimilation, de l'acculturation ou dans des aspects plus positifs, de la rencontre et du métissage...

Il faut alors se tourner vers un aspect plus concret de la notion, sa dimension politique.

\section{Indianité politique}

$\underline{\text { Retour à la table des matières }}$

En effet, on peut considérer que l'Indianité, telle qu'elle est mobilisée dans l'art contemporain, trouve ses racines dans la définition politique de la notion. L'Indianité est née avec l'essor de ce que l'on a nommé le panindianisme, dans les années 1960, aux Etat-Unis et au Canada : c’est d'ailleurs alors qu'apparaît le terme "indianity”.

Il s'agissait de "faire renaître l'Indien" 6, car les identités tribales étaient menacées par les processus d'assimilation mis en place par les gouvernements, notamment à travers les pensionnats indiens, les residential et industrial schools au Canada et indian ou boarding schools aux États-Unis. Ces pensionnats visaient à "blanchir” les enfants amérindiens, à les évangéliser et les “civiliser” en même temps, à travers la répression systématique de la pratique des langues et coutumes amérindiennes ${ }^{7}$.

mais ont tout de même voulu figurer sur le registre des Affaires Indiennes. Enfin, il existe aussi les "Indiens sans statut », qui n’ont aucun droit et reconnaissance. Cependant, ces trois catégories, sans cesse dénoncées comme fictives et rigides, ne sont en rien représentatives de toutes les personnes d'ascendance amérindienne, déclarées ou non et de leurs rattachements communautaires, de même qu'elles ne répondent pas aux problèmes des populations métisses.

6 Rostkowski Joëlle, Le renouveau indien aux États-Unis, L’Harmattan, Paris, 1986.

7 Grant Agnès, No End of Grief. Indian Residential Schools in Canada, Pemmican Books, Winnipeg, 1996 et Pettipas Katherine, Severing the Ties that Bind. 
À partir des années 1950, les Etats-Unis et le Canada vont partager le même point de vue sur le " problème indien " : lui trouver une solution définitive afin d'en finir avec les spécificités du statut des Indiens et achever enfin de les assimiler. Aux États-Unis est ainsi votée le 1er août 1953, la Termination Policy (politique de liquidation), à travers la résolution 108, qui met officiellement fin à la responsabilité fédérale en matière d'affaires indiennes, et doit aboutir à terme à la disparition des réserves.

Avec les années 1960-1970, en grande partie d'ailleurs en réaction vis-à-vis de cette " politique de liquidation », va naître un mouvement contestataire indien ${ }^{8}$ parallèle, mêlé, utilisé ou inspiré, selon les moments et les organisations considérées, tout à la fois par les mouvements noirs pour les droits civiques et par les mouvements pacifistes contre le Vietnam.

C'est notamment au Canada en réaction au Livre Blanc, promulgué en 1969 par Trudeau, que va débuter le mouvement de la « renaissance indienne ». Le Livre Blanc visait en effet à ce que les Amérindiens deviennent des citoyens canadiens à part entière : il fut reconnu comme un louable projet, mais aux méthodes inacceptables pour les communautés autochtones. Il s'agissait de retirer la Loi sur les Indiens de 1876 et de faire disparaître les réserves, à l'image de la Termination Policy américaine. Le projet fut perçu comme une tentative supplémentaire d'assimilation et de négation des droits territoriaux, sous couvert de bons sentiments. Les Indiens de l'Alberta, réunis en conseil, publièrent ainsi en 1970 une contre proposition nommée le Livre Rouge.

Government Repression of Indigenous Religious Ceremonies on the Prairies, The University of Manitoba Press, Winnipeg, 1994.

8 Marienstras Elise, La résistance indienne aux Etat-Unis du XVIème au XXe siècle, Gallimard, Paris, 1980. 
Dans ce mouvement, tant sur la scène américaine (États-Unis et Canada), que sur la scène internationale, les Amérindiens vont redevenir «visibles », montrer qu'ils sont là, qu’ils ont survécu, et ce, avec force costumes, plumes et actions spectaculaires, les plus célèbres demeurant peut-être les actions de l'American Indian Movement, comme la " prise » d'Alcatraz en 1969, le siège du tristement célèbre Wounded Knee en 1973 ou encore la Longue Marche vers Washington en $1978{ }^{9}$. En arrière-fond à ces manifestations spectaculaires, se met en place un élan de fédération des nations indiennes, dans des organisations de portée fédérale, nationale, puis internationale.

En Saskatchewan (Canada), les organisations panindiennes naissent dès la fin de la seconde guerre mondiale, avec la Saskatchewan Indian Association en 1944, The Union of Saskatchewan Indians en 1946, puis en 1970 The Federation of Saskatchewan Indians. Au Canada naît en 1968 la puissante National Indian Brotherhood, qui entretient dès ses débuts des liens étroits avec le National Congress of American Indians aux Etats-Unis. Cette dynamique débouchera sur la rencontre essentielle à Standing Rock (Etats-Unis), en 1974, réunissant pour la première fois des représentants issus de 98 nations indiennes d'Amérique du Nord et Latine.

Je ne peux que résumer brièvement ici tout l'essor et l'impact politique du Red Power, déjà profondément développé et analysé dans de

9 L'occupation de l'île d'Alcatraz par l'AIM, en 1969, visait à mobiliser l'opinion publique américaine sur la condition indienne. L'île transformée en prison (baie de San Fransisco) devenait le symbole des réserves. Les militants de l'AIM, habillés de costumes traditionnels spectaculaires, proposèrent d'acheter Alcatraz "pour vingt-quatre dollars, payables en verroterie et cotonnade rouge, conformément au marché passé avec les Blancs trois cents ans plus tôt pour l'achat de l'île de Manhattan”. Les deux autres manifestations étaient des commémorations-happenings du massacre de Wounded Knee 1890, et de la Longue Marche aussi appelée Chemin des Larmes (1838), déportation des tribus indiennes et notamment des Cherokee d'est en ouest vers les réserves. Dans le cadre de la marche sur Washington, le chemin se faisait $a$ contrario, d'ouest en est (de San Fransisco à Washington), marquant là le temps de la reconquête. 
nombreux ouvrages de qualité ${ }^{10}$. Ce qui va nous intéresser davantage est de comprendre comment ce qui a été nommé "résurgence » ou « renaissance » identitaire, trouve également un prolongement dans le renouveau de l'art. Car c'est bien dans ces mouvements que naît peutêtre ce qui est de l'ordre d'un sentiment plus nouveau que résurgent : celui d'être indien, plus que d'être lakota, cree ou encore blackfoot. Un sentiment d'appartenance fondé sur des traits communs entre les cultures amérindiennes, ce que l'on a d'abord appelé le "panindianisme », puis l'indianité (indianity) ${ }^{11}$ et qui s'affirme à cette époque, permettant de dépasser les clivages locaux ${ }^{12}$ pour accéder à une plus grande assise politique internationale.

Ainsi l'indianité qui émerge à cette époque est avant tout une conscience d'appartenir à une « communauté », qui en tant que telle a droit à une reconnaissance officielle. Ce sentiment d'appartenance ne va pas seulement s'appuyer sur l'attachement à la terre et sur la revendication des droits (respect des traités, statut juridique, autodétermination...), elle va également se forger dans la pratique de cérémonies inter-tribales et l'échange de styles esthétiques. Ces styles ne pourront plus être désignés comme tribaux, mais bien comme « indiens » et non plus cree, lakota ou navajo par exemple, dès lors que des artistes, cree par exemple, se mettront à utiliser des motifs ou des techniques lakota, et ainsi de suite. Le continent Babel (j'emprunte

10 Par exemple, Nagel Joane, American Indian Ethnic Renewal. Red Power and the Resurgence of Identity and Culture , Oxford University Press, Oxford, 1996.

11 Rostkowski Joëlle, L’indianité, contextes et perspectives, Presses Universitaires de Nancy, 1988.

12 L'Amérique avant la colonisation était un continent Babel, aux langues et cultures extrêmement diverses, où les rivalités entre tribus et confédérations étaient nombreuses. Elles furent même parfois exacerbées par la colonisation et les alliances successives avec les nations colonisatrices. L'exemple peutêtre le plus connu : les guerres de fourrure du XVIIème siècle dans la région des Grands Lacs canadiens, pendant lesquelles Français et Anglais ont su jouer des rivalités économiques et politiques des Hurons et des Iroquois, ennemis traditionnels. Pour en savoir plus : Trigger Bruce, Les Indiens, la fourrure et les Blancs, Boréal Seuil, 1992. 
l'expression à Philippe Jacquin) que je décrivais plus haut va ainsi progressivement s'unifier autour de ces traits communs. On va également observer à l'époque ce qui sera appelé le «retour aux traditions », essentiellement tourné vers la pratique religieuse, la Danse du Soleil revenant en force dans les années 1960-1970, ainsi que le recours aux hommes-médecine.

Par défi, par imitation aussi, les grands leaders indiens utilisent alors tous les stéréotypes de l'Indien de cinéma pour asseoir l'impact de leurs discours sur les mémoires et les imaginaires des "Blancs ». Mais aussi par retour d'un goût pour ce qui est " authentiquement rouge ", les jeunes Amérindiens vont porter à nouveau les cheveux longs, les vestes brodées ou encore les mocassins. Certes, les critères de la mode "hippie» de l'époque favorisent ce regain pour "l'ethnique », au sens commun du terme, mais l'impact est je crois plus profond. Avec le Red Power, ce ne sont pas uniquement les consciences des «Blancs » qui ont été éveillées, ce sont aussi celles des Amérindiens. Pour la première fois depuis des années, on pourrait même se risquer jusqu'à dire des siècles, ils vont devenir le centre d'une attention dont le but n'est pas de les détruire, de les critiquer ou de les assimiler. Ils vont même parfois devenir des sortes d'égéries de la lutte sociale (pour les pacifistes et les « ancêtres » des alter mondialistes par exemple) ou de la conscience écologique ou spirituelle (pour les adeptes du New Age entre autres), selon ceux qui les idéaliseront, voire les utiliseront. Les Amérindiens vont alors trouver des ailes dans ce nouveau regard porté sur eux et sortir de la torpeur et de l'abattement qui avait fini par les rendre muets. Certes ce contexte les a favorisés, mais ils ont su également saisir l'occasion de se dresser à nouveau et d'utiliser des outils qui n'étaient pas a priori destinés à leur offrir une émancipation, comme les médias, l'éducation, la justice.

Avec le panindianisme émerge donc un consensus indien, se fondant essentiellement au départ dans les milieux urbains (des Indiens de différentes communautés, déracinés, en recherche de « communauté ») et en anglais. Cette Indianité met donc en évidence, plutôt que 
les différences, les éléments communs transtribaux comme le respect des aînés, la tradition orale, la défense de l'environnement, le rapport spécifique à la Terre Mère, les cérémonies comme la Danse du Soleil, la cérémonie de la pipe, le give-away ${ }^{13}$, les loges à sudation... On est alors en passe de construire un nouveau rapport de sens, dans l'émergence d'un “peuple indien” versus “les peuples amérindiens”. Dans une certaine mesure, il s'agit là d'une acculturation nécessaire. Elle passe par l'adoption de traits non-indiens comme l'anglais, les médias et méthodes juridiques d'action, qui deviennent progressivement "typiquement” indiens. La langue, adaptée dans un phrasé anglais très particulier et l'invention d'idiômes spécifiques ${ }^{14}$, une surmédiatisation et stéréotypisation volontaire et instrumentalisée par les mouvements indiens eux-mêmes (la prise d'Alcatraz que j'évoquais plus haut en est un très bon exemple), ainsi qu'une utilisation effrénée du droit 15 , aboutissent ainsi à la construction imaginée d'une "nation" (pensons au jeu de cricket chez Appaduraï ${ }^{16}$ ).

13 Les give away sont des cérémonies de don organisées dans les contextes familiaux, afin de remercier les esprits et la famille, lors d'évènements importants (naissance, anniversaire, initiation, mariage, décès...).

14 Voir par exemple chez Silver Shirley et Miller Wick, American Indian Languages. Cultural and Social Contexts, University of Arizona Press, Tucson, 1997.

15 Rien qu'aux Etats-Unis, entre 1946, date de sa création et 1979, la Commission sur les revendications indiennes (Indian Claims Commission), qui existe également au Canada, a tranché quelques 300 litiges territoriaux et attribué 640 millions de dollars d'indemnités à différentes tribus ou individus... Le budget annuel de cette commission au Canada, pour l'année 2005/06, était de 6.9 millions de dollars...

16 Appaduraï Arjun, Après le colonialisme. Les conséquences culturelles de la globalisation, Payot, Paris, 2001. Le jeu de cricket est envisagé par Appaduraï comme l'exemple même d'une décolonisation réussie. Le cricket, symbole par excellence du monde anglais victorien de la colonisation, "forme culturelle dure”, pour reprendre l'expression d'Appaduraï, qui aurait dû résister à l'indigénisation, est paradoxalement devenu l'incarnation de l'Inde ellemême. Il ne s'est pas simplement indigénisé, mais par volonté politique et économique, conjuguée au désir des foules et à la médiatisation, il est devenu un sport national et même nationaliste, victoire de l'Inde sur l'ancien colonisateur... De même le droit anglais et la langue anglaise, transformés et adoptés par les Amérindiens, sont devenus les armes mêmes de leur émancipation vis- 


\section{Indianité entre symboles et stéréotypes}

\section{$\underline{\text { Retour à la table des matières }}$}

L'Indianité apparaît donc difficile à définir, oscillant entre une définition quasi ontologique d'un "être indien”, essence définie par l'extérieur (avec toutes les dérives ethnocentriques voire racistes possibles) ou par ailleurs par elle-même ; et une définition contextuelle, historique et dynamique...

L'Indianité longtemps incarnée dans le cinéma hollywoodien par exemple, était une indianité avant tout fantasmée par le regard occidental, une image d'Epinal qui concentrait l'essence du "noble" ou du "mauvais" sauvage, et finalement nous renseignait bien plus sur le point de vue occidental de l'époque, que sur le point de vue amérindien.

La mise en scène de cette indianité de clichés, par les Amérindiens eux-mêmes, dans un contexte de revendications politiques (les images bien connues des années 1960 que j'évoquais plus haut), constitue une prise de pouvoir vis-à-vis de cette image imposée par l'extérieur. Il s'agit donc ensuite de l'instauration d'un jeu très complexe entre identités et images, qui se met en scène dans l'alternance.

Parfois il faut se conformer à ces stéréotypes, dans la nécessité d'obtenir une reconnaissance des siens qui paradoxalement passe, elle aussi, par le relais du fantasme et de l'adéquation au désir et stéréotype de l'autre ; parfois il faut s'en détacher, pour mieux dénoncer son caractère enfermant et réducteur.

à-vis des gouvernements canadiens et américains, dans un processus semblable à ce qu’Appaduraï appelle le “dialogue de la décolonisation”. 
En effet, celui qui porte les tresses et le collier de perles (ou mieux de piquants de porc-épic 17 ), repères symboliques d'une indianité stéréotypée, aura l'air d'un « vrai indien » et sera reconnu comme tel non seulement par l'extérieur, mais aussi par les siens. Cependant, ces “stigmates" 18 de l'indianité, s'ils sont nécessaires tout particulièrement à cette époque, ne seront pas suffisants pour la communauté. Si cet individu n'aime pas le pain frit, ne participe pas aux sweat lodge, vit en ville et ne retourne jamais sur la réserve, il sera vite traité de « red apple » : rouge à l'extérieur, blanc à l'intérieur. La question est donc de partager un ensemble de références et de pratiquer les attitudes considérées comme communes aux «Indiens » en général et qui les distinguent vis-à-vis de l'extérieur, des Blancs... On retrouve bien là le caractère principal de l'ethnogénèse pour l'anthropologie culturelle américaine : la consolidation d'une identité dans l'opposition et la démarcation vis-à-vis d'un groupe dominant.

L’adoption de ces références se joue dans une négociation complexe entre stéréotypes, valeurs internes et externes au groupe, et traits communs cosmologiques transtribaux. Ainsi, la croyance aux esprits, la pratique du rêve, l'interconnexion du vivant sont par exemple des principes fondamentaux partagés par un grand nombre de tribus amérindiennes, qui reconnaissent elles-mêmes cette parenté 19 .

Les tentatives d'incorporation de l'Indianité par les dits "Blancs” sont d'ailleurs à cet égard très intéressantes, car très paradoxales :

17 La broderie en piquants de porc-épic est la première technique indienne de décoration des possessions. Elle prévalait avant l'introduction des perles par les Européens au 17ème siècle. Elle apparaît donc comme culturellement plus " pure »...

18 Au sens de Goffman Erwin, Stigmate, les usages sociaux des handicaps, Paris, Ed. de Minuit, 1963.

19 Voir par exemple Battiste Marie \& Youngblood James, Protecting Indigenous Knowledge and Heritage. A global Challenge, Purich Publishing, Saskatoon, 2000. 
l'exemple célèbre de Grey Owl 20 met en évidence cette complexité. Adoptant tous les codes, partageant toutes les valeurs de l'Indianité telle que définie et prônée par les Amérindiens eux-mêmes, tout d'abord porté aux nues comme premier défenseur de l'écologie, l'anglais Archie Belaney, de son "nom indien” Grey Owl, sera finalement rejetté comme imposteur et mystificateur à l'annonce de sa non indianité de sang...

Il semble donc parfois qu'il ne suffise pas de partager des codes, attitudes et valeurs, mais qu'il faille aussi partager le code génétique, au moins en partie, pour pouvoir se réclamer indien et porteur d' Indianité. On en vient ici progressivement à dégager à nouveau une dimension substantiviste, une certaine idée de l'authenticité. La distinction entre l'essence et la construction de l'être resurgit dans toute sa complexité car les deux phénomènes ne sont pas forcément dissociables. Nous verrons cependant que, dans le cas de l'art contemporain de l'Indianité, est inventée une authenticité non plus tribale et attachée à un pourcentage de sang indien coulant dans les veines de l'artiste, mais imaginée dans une dimension transnationale, et sur des critères d'appartenance très larges. Les paradoxes de l'identité, mais surtout des définitions identitaires, ne manquent pas. L’Indianité dans l'art contemporain amérindien apparaît même comme un dépassement de la notion d'indianité telle qu'elle s'est définie politiquement dans les années 60-70. Elle révèle les tensions et transformations subies et initiées par les groupes sociaux comme les individus, dans le nouveau contexte de mondialisation. Il semble en effet que la notion d'indianité puisse à nouveau être redéfinie et mise en mouvement par les acteurs, que le processus (peut-être d'ethnogénèse) soit encore en construction, dans une transnationalité et transculturalité élargie.

20 Deloria Philippe J., Playing Indian, Yale University Publishing, Yale, 1998 et Francis Daniel, The imaginary Indian. The Image of the Indian in canadian Culture, Arsenal Pulp Press, Vancouver, 1992. 


\section{L'Indianité comme dépassement des catégories de l'art tribal vers l'art « universel »}

$\underline{\text { Retour à la table des matières }}$

Dans le contexte de l'art, puisque c'est celui qui sert de terrain d'expérimentation à cette réflexion, la valeur d' " authenticité » que nous venons d'évoquer peut être envisagée comme une valeur ajoutée à l'oeuvre. En effet, la labellisation de l'Indianité est utilisée dans le contexte muséographique comme argument de vente et d'exposition de l'oeuvre, dans les musées d'ethnographie certes, mais aussi dans les nombreuses galeries dites primitivistes, qui ont fleuri en Europe depuis l'engouement des surréalistes pour les arts extra-occidentaux (années 30). L'ethnicité de l'oeuvre semble ainsi forger son originalité et plus encore sa valeur, son intérêt même : c'est parce qu'il est possible et même nécessaire d'associer à une oeuvre un commentaire ethnologique qu'elle devient intrigante et digne d'exposition...

Dans la démarche inverse, qui existe elle aussi, mais de façon beaucoup plus récente (fin des années 60, avec la décolonisation), on voit au contraire émerger une volonté de dés-ethniciser l'oeuvre et de récuser la notion même de l'authentique, qui nierait aux artistes non occidentaux comme à leurs productions, un accès possible à l'histoire, au changement et donc à l'invention. L'authenticité est bien sûr, dans cette perspective, mobilisée dans son acception évolutionniste, en tant que mythe de la référence au pur, au stable, au préservé, à l'originel. Dés-ethniciser l'oeuvre et son auteur implique de ne pas fournir de notices biographiques ou explicatives au public, afin de rompre avec un cliché sur l'art non-occidental qui nécessiterait toujours un commentaire anthropologique pour être bien compris. 
Le cartel, symbole de la logique informative du musée d'ethnographie, apparaît alors non comme un outil pour une meilleure compréhension, mais au contraire comme l'instrument de l'intraductibilité des cultures. En effet, le cartel, s’il vise, par le recours au texte, une meilleure compréhension entre le public et l'oeuvre issus de cultures différentes, n'empêche pas et même parfois génère des aberrations de sens dues à la retranscription et à l'ethnocentrisme. Parallèlement, il tend à enfermer l'objet, et par procédé métonymique, la culture dont il provient, dans des frontières strictes de territoire et d'appartenance (on figure toujours autant que possible la provenance géographique, l'ethnie...). Par nécessité d’être simplifié et accessible au plus grand nombre, le cartel peut ainsi tendre à réduire également l'objet lui-même, son sens, sa portée, et même à brider alors l'interprétation libre qu'aurait pu en faire le spectateur. Ce n'est plus ce qui est vu qui fait sens, mais le contenu de l'étiquette.

Dans ce débat, nous nous situons au coeur d'une problématique qui fait rage depuis une quarantaine d'années en France et ailleurs, autour de la question de l'universalité du beau et de l'art ${ }^{21}$. Les réflexions muséographiques modernes oscillent ainsi sans cesse entre les deux points de vue, que Jean Jamin a nommé les “ethnologisants” et les "esthétisants". On ne cesse de réinterroger le statut de l'objet, ainsi que les implications éthiques fondamentales que suppose son exposition (MEN 2002) ${ }^{22}$.

21 Voir par exemple le numéro de Terrain consacré au Beau (Terrain n³9, mars 1999), ainsi que la démarche d'exposition, centrée autour de l'analyse transformationnelle de Lévi-Strauss, proposée par Emmanuel Désveaux au musée du Quai Branly. Désveaux Emmanuel, “Un guide sur la question de l'autre”, in "Lévi-Strauss, l'ethnologie ou la passion des autres”, Magazine Littéraire, Hors série $n^{\circ} 4,2003$, pp : 48-51.

22 MEN (collectif), Le musée cannibale, GHK Editeurs, Neûchatel, 2002. 


\section{Le paradoxe des identités}

\section{$\underline{\text { Retour à la table des matières }}$}

Dans ce contexte, l'Indianité (et non la tribalité, puisqu'il s'agit bien ici de se distinguer des Autres, en tant que référence élargie) est mobilisée comme une stratégie identitaire par les artistes contemporains face à l'art dit « occidental » (euro-canadien, euro-américain), tout en utilisant cependant les codes et médiums internationaux (et donc en grande partie, occidentaux) dans les oeuvres elles-mêmes. On voit ainsi en action un processus de mimétique avec l'art occidental, qui s'avère pertinent dans la mesure où l'art occidental s'affiche et se vend aujourd'hui comme un art hors frontières, un "art global” ou "art mondial”...

Pour les artistes amérindiens, il s'agit donc d'un double défi, être à la fois un autre et un "même", identifiable, entre reconnaissance et distinction. Cette dynamique s'inscrit donc plus largement dans un contexte général de “jeu” entre les référents identitaires et de négociation d'une image et d'une place au sein du monde l'art contemporain.

Pourquoi une telle dynamique ? Parce qu'il faut bien partir d'un constat, qui rejoint les problématiques muséographiques exposées plus haut. Bien souvent les acheteurs " occidentaux », ne sont intéressés par l'art non-occidental que s'il est distinctement (visiblement) reconnaissable en tant que tel, c'est-à-dire comme « différent », être "différent” étant encore souvent synonyme d'exotique et de primitif. Ce constat nous amène à réfléchir sur le fait que les artistes indigènes qui utilisent justement des formes et codes, des styles, pensés comme relevant de l'art occidental, sont difficilement identifiés, reconnus et donc vendeurs. 
Il apparaît alors une flagrante contradiction entre les désirs des artistes d'être reconnus internationalement, ce qui suppose d'adopter des codes qui finalement ne leur apparaissent plus comme occidentaux mais bien internationaux (et nous le verrons issus de la rencontre et de l'interaction entre les cultures), et les représentations du public, toujours en recherche de l'exotique, de l'Autre. Les travaux des artistes contemporains apparaissent alors aux yeux du public comme « dénaturés », " acculturés », en un mot moins authentiques.

Dans le même temps, et c'est là qu'apparaît le système dans toute sa duplicité, l'artiste indigène qui ne créerait qu'avec des codes dits " indiens » serait immédiatement enfermé dans la catégorie "arts traditionnels" et n'accèderait rigoureusement pas au même type de marché et au même type de reconnaissance : au marché des arts dits "premiers", mais pas à celui de l'art contemporain international, et à des lieux d'exposition comme les musées d'ethnographie, mais pas aux musées ou galeries d'art contemporain. Dans les deux cas, l'identité et ses définitions se révèlent source de complexité et de difficultés.

Le concept d'hybridité proposé par James Clifford 23 relève de cette contradiction. Clifford, souhaitant célébrer les « contaminations culturelles », dans un monde où le surréalisme (transformation, hybridation et métamorphose) n'est plus marginal et atypique, mais où c'est le concept de produits purs qui devient anormal, amène à considérer les hybridations non plus comme des aberrations, mais comme la normalité dans l'ordre quotidien (et universel, global) des choses. Clifford imagine :

« des manifestations qui privilégient les productions « inauthentiques » et « impures » de la vie tribale passée et présente, des expositions radicalement hétérogènes par leur mélange de styles, des expositions qui se situent dans des conjonctures multiculturelles particulières, des expositions où la

23 Clifford James, Malaise dans la culture. L'ethnographie, la littérature et l'art au XXème siècle, ENS Beaux-Arts, Paris, 1996, p. 212. 
nature reste « non naturelle », des expositions dont les principes d'incorporation soient ouvertement contestables. »

Cependant, une critique avancée par certains serait que l'hybridité tend alors à masquer les hétérogénéités persistantes et revendiquées : célébrer une idée globale de la différence peut nuire à la compréhension des différences « locales » en construction. L'hybridation « totale » peut alors amener à reconduire ce que l'on combattait : l'essentialisme. C'est ici une critique apportée par certains artistes contemporains et anthropologues de l'art comme Jeremy MacClancy : certains critiques ou conservateurs dans les musées s'autoriseraient par ce discours sur l'hybride à ne mobiliser que leurs propres codes et capacités à reconnaître la différence culturelle, sans avoir plus besoin d'un recours au discours de l'artiste et de l'oeuvre elle-même ${ }^{24}$. Certains artistes rejettent également cette idée d'une hybridité ou d'un métissage dans la mesure où cette catégorisation est souvent employée de manière fixiste : elle renvoie à un produit figé de la rencontre entre deux cultures. Or beaucoup d'artistes amérindiens, qui utilisent des codes dits “occidentaux" et des codes dits "indiens", soulignent le caractère dynamique d'un perpétuel flux artistique entre les cultures, où aucune d'entre elles ne domine et où les tensions sont également permanentes, chez Edward Poitras ou Jimmie Durham par exemple.

Une oeuvre telle que "Big Iron Sky” (1984) d'Edward Poitras, artiste métis lakota originaire de la Saskatchewan ${ }^{25}$, utilisant le support de l'installation, forme artistique récente et occidentale dans ses origines, met en scène la cohabitation des mondes. Mélangeant des matériaux “naturels" souvent reliés au primitivisme et à l'Indien (os, bois, cuir, crânes de chevaux) et des matériaux "technologiques" et "blancs”, comme la fibre carbone et les câbles en acier, le sujet représenté dans une complète modernité est cependant, et sans aucun doute

24 MacClancy Jeremy (ed), Contesting Art. Politics and Identity in the modern World, Berg, Oxford, 1997.

25 La Saskatchewan est une province du centre ouest du Canada, où j'ai effectué des recherches de terrain depuis 2001. 
possible, identifiable comme "Indien", dans cette Indianité que je m’efforce ici de mettre en lumière.

Une oeuvre comme "Red Man watching White Man trying to fix Hole in the Sky" (1990) de Lawrence Paul, artiste salish (Côte NordOuest), met également en évidence cette nutrition réciproque et réappropriation des styles culturels les uns par les autres, à travers une représentation à l'esthétique très imprégnée du surréalisme de Dali, mêlé sans discontinuités avec des motifs typiques de l'art tribal de la Côte Nord-Ouest. Quant au sujet de l'oeuvre, il est essentiel dans le mouvement de l'Indianité, et reflète bien des valeurs mises en évidence dans le panindianisme : le militantisme politique et écologique.

Les deux artistes allient ainsi au coeur de leur création leurs identités tribales, à travers les codes esthétiques lakota et salish mobilisés dans l'emploi de certains motifs et couleurs; leurs appartenances transtribales avec l'appel à des motifs et codes issus d'autres références culturelles amérindiennes entrant en résonance avec les premières, mais aussi à travers la mise en scène de thématiques panindiennes comme celle de la Terre Mère ; et enfin leur désir d'internationalité, de reconnaissance universelle et d'ancrage dans la modernité, avec l'utilisation de médiums et supports internationaux.

En continuant dans le sens de cette dynamique d'interpénétration des styles esthétiques, mais dans le mouvement géographique inverse, il faut rappeler que les artistes occidentaux modernes se sont également abondamment nourris de styles artistiques extra-occidentaux, se proclamant même du " primitivisme », comme Picasso, Georges Braque ou Max Ernst, et que c'est précisément cette rencontre qui a construit "l'art moderne".

Par ailleurs, les artistes amérindiens soulignent aussi que la « tradition », telle qu'elle est envisagée dans la perspective amérindienne, n'a rien de fixiste, qu' elle est au contraire fondamentalement ancrée dans une capacité d'adaptation et d'intégration de la nouveauté. Jimmie 
Durham, artiste et critique d'art cherokee travaillant à Berlin, dit à ce sujet :

« Les traditions existent et les communautés indiennes les préservent. Et l'une des plus importantes est le dynamisme. Le changement constant - au sens d'adaptabilité, d'intégration de nouvelles façons de faire et de nouveaux matériaux - est une tradition que nos artistes glorifient particulièrement, s'en servant pour faire évoluer et renforcer nos sociétés. » 26

Il s'agit d'ailleurs là d'une spécificité de l'Indianité : non fixée et non résolue ou apaisée, elle est mobilisée par les artistes dans des oeuvres où la tension, entre les cultures et leurs " influences ", est très forte, palpable. On voit sur la toile se déchirer, puis se joindre et se séparer encore les identités à échelles multiples de l'Indien : tribale, transtribale, nationale, humaine, etc...

La grande question pour les artistes contemporains amérindiens est finalement la reconnaissance : du public, certes, mais également (et peut-être avant tout dans les discours de nombre d'entre eux), de l'establishment international, de l'histoire mondiale de l'art. Etre dans les manuels, les encyclopédies, exister à part égale et entière, et bien sûr affirmer que l'on est pas mort, quand les créations extra-occidentales sont presque toujours confinées dans les musées d'histoire, les musées de la mémoire et du passé.

L'Indianité étant transnationale et transtribale, elle apparaît comme un recours légitime et approprié, puisqu'il s'agit d'en appeler à la scène internationale. Comme dans le contexte politique, la fédération audelà des appartenances tribales permet une plus grande lisibilité du mouvement, un poids plus considérable - l'union fait la force -, et permet aussi de montrer la volonté de sortir du ghetto de l'identité locale afin d'être soit aussi part du "grand mouvement du monde”...

26 Durham Jimmie, A certain Lack of Coherence : Writings on Art and Cultural Politics, Kala Press, Londres, 1993. 
Quand les revendications spécifiquement locales de telle ou telle tribu pour sa terre apparaissent comme des vestiges du passé, comme un replis vers la tradition - de ce point de vue envisagée comme passéiste et fixiste - ; être moderne c'est échapper à la terre, à l'espace et au temps comme à sa carte d'identité.

\section{Art et dépassement des catégories}

$\underline{\text { Retour à la table des matières }}$

Ainsi, les artistes de l'Indianité cherchent à dépasser les clivages entre art occidental ou non-occidental, local ou international, tout en conservant une spécificité indienne. Un bon exemple de cette démarche et de son caractère novateur, est la performance d'artistes nommée INDIANacts ${ }^{27}$, qui eut lieu pendant trois jours à Vancouver, au Canada les 29, 30 novembre et 01 décembre 2002. Cette manifestation se situait dans le mouvement dit de " l'art action amérindien », et avait pour perspective la rencontre et la transgression de chacun des deux styles mis en présence : d'un côté la "performance " médium très couru dans l'art contemporain le plus pointu, sur la scène internationale et occidentale, et de l'autre le story telling, la performance amérindienne « traditionnelle ».

Était présent par exemple le groupe des High Tech Storytellers, collectif d'artistes amérindiens : ils se situent au carrefour des deux démarches citées, ni dans l'une ni dans l'autre, mais dans une forme « rencontrée », imaginée de l'une et de l'autre. Des artistes se mettaient eux-mêmes en scène, entre haute technologie multimédia et "ra-

27 «INDIANacts » : ce nom joue avec l'Indian Act, la fameuse Loi sur les Indiens que j'évoquais plus haut. 
contage” 28 (storytelling) : voici le résumé proposé au public de la manifestation INDIANacts :

"L'événement fusionnera avec pertinence non seulement des performances mais encore de l'art action en dialogue avec la vidéo et l'art web pour ajouter à "lindiscipline" des conférenciers, quelques-uns se métamorphosant eux-mêmes en conférence/performance. Ajouter à cela ces moments de synthèse, de questions puis les prolongements de rencontres hors colloque, dans des ateliers et logis d'artistes, à la Maison Longue, lors d'expositions et ce, de la première soirée combinant expo et perfo à la Grunt Gallery jusqu'au Cabaret festif hybride en finale chez Western Front. Pendant trois jours, l'utopie maintes fois exprimée d'osmose entre en vie de l'art en actes a circulé. L'amérindianité artistique active vraiment le sentiment de communauté vivace, humaine et intelligente.”

Ces artistes cherchent ainsi à inscrire leurs expériences individuelles au sein d'une culture singulière, toujours en transformation, mais en même temps s'opposent aux enfermements et exclusions, au communautarisme. Dans le concept d'Indianité, ils recherchent un principe d'humanité, ce que Tvetan Todorov nomme un «humanisme critique » ${ }^{29}$, c'est-à-dire un humanisme avec pour postulat commun le refus des déterminismes, mais qui ne se départit pas pour autant d'une appartenance, culturelle ou autre. Etre reconnu à part sans s'enfermer, voilà le défi relevé par ces artistes...

Ils ont donc plus d'un point commun : une éthique de la reconnaissance, mais aussi une grande prégnance dans leurs oeuvres de conflits non résorbés, où leur réalité quotidienne, individuelle et sociale, est avant tout marquée par la négociation (des identités, des droits, des libertés...) comme en témoignent par exemple les oeuvres de Jane Ash Poitras, Bob Bowyer, Edward Poitras, Harry Fonseca, Lance Belan-

28 Il s'agit de la traduction officielle du terme de storytelling, dans la terminologie française canadienne.

29 Todorov Tzvetan, Nous et les autres. La réflexion française sur la diversité culturelle. Ed du Seuil, Paris, 1989. 
ger, ou encore Jimmie Durham ${ }^{30}$... Leurs productions mettent en scène un affrontement, une " discussion » entre les matériaux, les styles, révélant l'affrontement dans leur quotidien du «monde blanc » et du « monde rouge ».

Par exemple, le fameux tableau de Harry Fonseca, artiste maidu (Sud-Ouest, Etats-Unis),“When Coyote leaves the Rez' : Portrait of the Artist as a Young Coyote” (1980), met en scène un jeune Indien des années 1970-80.

Cet autoportrait, comme son nom l'indique, porte l'influence de la littérature occidentale (référence explicite au roman de James Joyce, "Portrait de l'artiste en jeune homme"). Il présente un coyote aux airs décontractés, fumant une cigarette, tout habillé de cuir noir, à la façon de Fonzie, le héros mais mauvais garçon de la série américaine Happy Days, ou plus récemment d'un jeune "loubard” en perfecto. L'artistecoyote "quitte la réserve" pour venir en ville, où il incarne l'image universelle de la jeunesse "sauvage”, qui dérange les âmes bien pensantes, à l'instar d'un Marlon Brando dans les sixties ou plus près de nous d'un Renaud dans la révolte des banlieues des années $80 .$.

L'artiste brouille les référents identitaires (entre occident blanc et modernité amérindienne), tout comme les catégories du vivant (entre animal et humain) reprenant là une démarche exemplaire en regard des catégories cosmologiques amérindiennes. En effet, Coyote est la figure par excellence du mythe, mi-homme, mi-animal, voyoudémiurge incarnant toutes les ambiguïtés de l'âme et de l'existence humaine ${ }^{31}$. Fonseca synthétise ici, tant dans la forme que dans le fond, le coeur des thématiques chères aux artistes de l'Indianité.

30 Pour voir des oeuvres de ces artistes réunies : MacMaster Gerald (ed), Indigena. Contemporary Native Perspectives in canadian Art, Craftsman House, New-York, 1992.

31 Coyote est le trickster ou héros décepteur : celui qui joue des tours, et qui par ses erreurs donne forme et matière au monde. Il est aussi la figure de la transgression des interdits comme des frontières entre les catégories du vivant. Par- 
En effet, ces artistes se fédèrent autour du questionnement même de ce qui les rassemble, cette fameuse notion d'indianité. Leur question est : qu'est-ce que l'Indianité, et même, qu'est-ce que l'identité. Ces artistes ont des origines diverses, ils sont cree, cherokee, lakota, ojibway, métis... Ils ne revendiquent donc pas une appartenance territoriale ou politique, mais bien une appartenance imaginée, en construction et questionnement perpétuel.

La question est donc extrêmement complexe et les mobilisations très processuelles et variables. Tantôt c'est l'une ou l'autre des attitudes ou facettes identitaires qui est mise en avant, parfois chez un même artiste. Une anecdote me permettra d'éclairer cette situation d'ambiguïté.

La scène prend place dans un galerie d'art contemporain (MacKenzie Art Gallery), à Regina, Saskatchewan. L'Indianité est affichée, nécessaire, labellisée vis-à-vis de l'extérieur dans le titre même de l'exposition : "Regards on native contemporary Art". Les artistes viennent donc de toutes les régions du Canada et sont de toutes origines tribales. Cependant, ces différences ne sont volontairement pas distinguées dans les commentaires qui accompagnent les oeuvres : on est indien avant tout et c'est ce qui compte, Indiens d'aujourd'hui, de cette indianité née au coeur de la modernité (depuis les années 1960). Les cartels ne spécifient donc pas les origines tribales des artistes, seuls leurs noms figurent, avec les descriptions classiques des matériaux employés, la date et le titre de l'oeuvre, et parfois quelques explications sur la démarche de l'artiste.

Pourtant, au fil de la soirée, déambulant à travers les salles, je surprends des conversations, entre artistes ou entre visiteurs. Sans cesse reviennent les références à la tribalité de l'auteur de l'oeuvre : «C'est

fois humain, parfois animal, parfois un peu des deux. Voir par exemple chez Erdoes R. et Ortiz A., Et Coyote créa le monde, Albin Michel, Paris, 2000. 
très cree comme style. Oui je crois bien qu'il est cree, c'est sûr, tu as vu ces motifs »... Plus loin, même type de discours, mais cette fois-ci par aire culturelle, création anthropologique relayée et réappropriée par les communautés amérindiennes elles-mêmes : "Tout à fait du Sud-Ouest, avec ces couleurs, ces matières... »

On constate ici à quel point l'ancrage identitaire précis de l'artiste demeure nécessaire : le public le demande, comme à la lecture d'une oeuvre, l'autobiographie peut éclairer. Il semble difficile de résister à l'envie de classer, catégoriser, reconnaître non seulement l'auteur, mais peut-être la source de son inspiration : pourra-t-on reconnaître un style, une influence et l'expliquer alors par l'origine, la transmission. C'est de la même façon que l'on cherche sans relâche à reconnaître le père ou la mère dans l'enfant qui vient de naître, ressemblance supposée que l'on croit alors être une preuve tangible, une explication naturelle, quasi inévitable, des comportements ou traits physiques de l'enfant...

Un peu plus tard, une autre conversation vient brouiller les pistes. Deux artistes plaisantent face à l'oeuvre de l'un d'entre eux :

« Oh lala, tes petits triangles et tes marques, c'est très sioux quand même ! T'as pas honte de voler tes voisins, c'est quand même le comble de faire du sioux pour un métis-cree... » - Rires - « Ouais c'est clair, mais tu sais nous les Cree on a toujours été un peu jaloux des fiers emplumés! Y'en a toujours que pour eux !.. Non mais sans rire, les triangles, c'est aussi cree et puis de toutes façons, ce qui est sûr c'est que c'est indien ! »

Face ces modalités d'alternance, il existe également un courant dit « traditionnaliste », qui entend préserver et maintenir des formes artistiques de représentations des savoirs, où les sujets nécessitent alors d'être documentés et approuvés par les Aînés ${ }^{32}$. Cependant la majori-

32 Les Aînés sont consultés par certains artistes soucieux de vérifier l'adéquation de leur travail avec la nomenclature traditionnelle parfois existante : respect des codes de la figuration, emploi de motifs strictement affiliés dans leur ethnicité, choix des couleurs, autorisation dans les sujets traitables ou non... 
té des artistes contemporains tendent eux, à travers cette création dans le métissage et l'invention, à mettre à jour de nouvelles catégories classificatoires, cette fois-ci trans-tribales.

Des artistes parviennent également à allier et transcender les deux points de vue, comme Oscar Howe, artiste dakota (1915-1983) : il est reconnu comme artiste traditionnel au sein de sa communauté et pourtant il bouleverse les codes évoqués plus haut. Il utilise à la fois les exigences du style traditionnel, notamment en respectant le réalisme pictural, figuratif des œuvres ${ }^{33}$, mais introduit également les leçons du cubisme qu'il aménage pour donner du mouvement dans la représentation et l'agencement des couleurs.

Un autre innovateur important que l'on peut citer ici est Daphne Odjig, qui fonde en 1970 la Professional Native Indian Artists Incorporated, connue aussi sous le nom de «Indian Group of Seven » : une association qui combattit les politiques culturelles du Département des Affaires Indiennes canadien. Ce dernier tendait selon le Groupe à annihiler, par des stratégies marketing de formatage de l'indianité dans l'art, les spécificités individuelles des pratiques de l'art contemporain amérindien. Ces artistes cherchaient à souligner dans leur recherche une double dimension : l'appartenance à l'Indianité (à travers le partage de références et de valeurs) mais aussi la liberté individuelle. Nous sommes ici bien éloignés de l'artiste primitif tel qu’il était envisagé par exemple par Boas dans les débuts de l'anthropologie américaine,

33 La création artistique chez les Dakota suivait des normes strictes et notamment la dichotomie sexuée des styles : les hommes pratiquaient le style figuratif dans des oeuvres peintes et les femmes le style géométrique à travers la broderie en perles et piquants de porc-épic. Voir par exemple Desveaux Emmanuel, «Les Grands Lacs et les Plaines, le figuratif et le géométrique, les hommes et les femmes », Papers of the 24th Algonquian Conference, 1993. Cependant, certains exemples viennent contrecarrer cette dichotomie figée, voir Goyon Marie, « La logique du lien dans l’art du travail aux piquants des Amérindiens des Prairies ", Recherches Amérindiennes au Québec, volume $X X X V I, \mathrm{n}^{\circ} 1,2006$. 
c'est-à-dire comme simple répétiteur de la tradition, transmise presque immuable de génération en génération ${ }^{34}$.

\section{En conclusion}

$\underline{\text { Retour à la table des matières }}$

Ainsi le flux de la mondialisation et le changement d'échelle ont semble-t-il eu comme effet, bien plus que de «lisser » ou d'homogénéiser autour d'une culture blanche et occidentale, de démultiplier les possibilités de réappropriation des signes associés à la modernité occidentale dans des stratégies identitaires multiples. Ces signes vont alors fonctionner en liaison avec un répertoire mettant en oeuvre une toute autre historicité, celle des cultures "réceptrices". Dans ce contexte, l'art contemporain amérindien apparaît en effet particulièrement révélateur de cette dynamique.

Jimmie Durham encore, montre bien dans les propos suivants la construction du «nous » qui n'est ni tribal ni national, mais imaginé dans l'Indianité, face aux « autres » :

« Depuis 350 ans notre histoire est liée à la votre -les Blancs-. Elle est devenue étrangère, intenable, insoutenable et insupportablement fausse. Notre histoire est devenue un mensonge à l'intérieur de la votre. » 35

Il affirme aussi, mettant ainsi en évidence la perpétuelle tension, l'aller-retour incessant entre les déterminations identitaires présent chez les artistes de l'Indianité : « je suis un artiste cherokee qui tente de faire de l'art indien, qui soit considéré juste comme universel et

34 Boas Franz, L'art primitif, Ed. Adam Biro, Paris, 2003.

35 Jimmie Durham cité par Virginie Colinet, in MacClancy J.(ed), Contesting Art. Politics and Identity in the modern World, Berg, Oxford, 1997, p. 52. 
sans limites, au même titre que l'art de l'homme blanc » ${ }^{36}$. En utilisant le terme "universel” pour définir son processus créatif, Durham n'entend pas s'inscrire dans une culture “occidentale” et dominante, mais bien produire un art libre qui explore les cultures en interrogeant les enjeux de leurs relations.

C'est ainsi que dans son travail, alors qu'il est lui-même cherokee (sud-est des Etats-Unis), il réalise par exemple des décors pour un opéra, "Chief Joseph ». Mis en scène et écrit par un auteur allemand avec une partition dans sa langue et teintée de musique classique, l'opéra s'élaborait à partir de l'histoire du Chef Joseph, un chef NezPercé (centre du Canada, tribu très différente des Cherokee) charismatique, ayant combattu pour l'indépendance de son peuple en 1879. Cet opéra kistsh a été mis en scène tout d'abord en 2005 à Berlin, puis représenté internationalement. Les décors de Durham consistaient en une déclinaison des stéréotypes de l’Indien hollywoodien.

Finalement, c'est avec Appaduraï que l'on peut me semble-t-il bien penser cette Indianité élaborée par les artistes. En la considérant comme un ethnoscape, au sens où il envisage ce paysage : c'est-à-dire un paysage où la localité en tant que telle n'existe pas et est invention permanente. Ainsi ce sont les groupes qui produisent leur local dans un contexte donné (historique, économique, ici artistique), et non la pesanteur d'un territoire qui façonne le groupe comme tel. Ce ne sont plus les tribus et leurs territoires ou encore les aires culturelles qui déterminent les styles culturels que l'on est en droit d'adopter, mais bien les artistes qui reformulent sans cesse leurs appartenances se faisant ainsi écho d'une réalité sociale bien plus large. Ainsi, comme le souligne Appaduraï :

"Il faut considérer la modification de la reproduction sociale, territoriale et culturelle de l'identité de groupe. Les groupes migrent, se rassemblent dans des lieux nouveaux, reconstruisent leur histoire et reconfigurent leur projet ethnique. Dans ce processus, le "ethno" d'ethnographie prend un

36 Jimmie Durham in Virginie Colinet, opus cit., p. 53. 
aspect fuyant, non localisé, auquel les pratiques descriptives de l'anthropologie devront répondre. Dans le monde entier, les paysages d'identité de groupe -les ethnoscapes- ont cessé d'être des objets anthropologiques familiers : désormais, les groupes ne sont plus étroitement territorialisés, ni liés spatialement, ni dépourvus d'une conscience historique d'eux-mêmes, ni culturellement homogènes.” 37

La tribalité est en partie conçue comme un frein, empêchant de rejoindre une Indianité internationale et libératrice, revendiquant le droit à la "contamination culturelle” 38 . En effet, comme le souligne Paul Ardenne ${ }^{39}$, l'art contemporain international voit l'avènement de ce qu'il appelle une “esthétique de la contamination” à travers l'emploi généralisé du détournement, du collage ou encore de l’assemblage. Le métissage et l'hybridation sont à l'oeuvre dans des réalisations plastiques où cohabitent des référents issus d'origines diverses, où les genres sont démultipliés, où les territoires culturels stricts sont abandonnés : par exemple chez le visionnaire Marcel Duchamp, le premier à déplacer et détourner des objets banals et usuels comme le fameux urinoir renversé sur un socle et baptisé "Fontaine” (1917), où encore Christian Boltanski qui questionne l'ethnographie et la déplace dans l'art ("L'inventaire des objets ayant appartenu à une femme de Bois Colombes”, 1974). Ceci n'est pas anodin puisque les historiens de l'art datent les débuts du mouvement de l'art contemporain à la fin des années 1960, en pleine phase de décolonisation : les concepts et les certitudes éclatent et chacun est amené à se redéfinir, à entrer en négociation ou à cristalliser une identité dores et déjà obsolète.

C'est ainsi que “art contemporain” et "mondialisation”, notions difficiles à cerner et qui ne cessent d'épuiser leurs propres définitions, révèlent de nombreux points communs, revêtant toutes deux une "géographie variable": le métissage des genres esthétiques comme

37 Appaduraï Arjun, Après le colonialisme. Les conséquences culturelles de la globalisation, Payot, Paris, 2001, p. 91

38 Clifford James, Malaise dans la culture, ENS, Paris, 1996.

39 Ardenne Paul, Art, l'âge contemporain. Une histoire des arts plastiques à la fin du XXè siècle, Ed du Regard, Paris, 2003, p. 297. 
des identités, la multiplication des procédures et la diversité des supports (land art, body art, low art, performances, happenings, installations multimédia...) comme la multiplication et reformulation incessante des modalités possibles d'élaboration de la notion même d'"identité", ou encore l'explosion des anciennes catégories fixistes tel que "bon” ou "mauvais" goût, "vrai” ou "faux" 40, "authentique” ou “acculturé”, “traditionnel” ou “moderne”...

Ainsi, l'art contemporain amérindien semble s'inscrire totalement dans ce mouvement, au carrefour d'imaginaires négociés, où les catégories d'art occidental ou extra-occidental perdent sens au profit d'un changement d'échelle radical.

À l'échelle internationale, le concept d'ethnoscape permet de rendre compte de la production d'une identité de groupe (d'une ethnogénèse) fondée sur certaines images, sur un "paysage” au sens d'un univers mental partagé. Ce sont les valeurs et pratiques auxquelles je faisais référence comme « identifiants » et qui imprègnent sans cesse les thématiques des oeuvres produites : la croyance en la Terre Mère et la conscience écologique, le partage de valeurs de solidarité et d'entraide, le respect des Aînés, des pratiques religieuses et sociales comme la Sundance et la sweat lodge, comme la conscience politique et le partage d'identités construites en tensions perpétuelles.

Enfin, on voit bien que la construction de ce paysage se fonde autant sur la mobilisation de la mémoire, de ses productions nostalgiques et parfois idéalisées (le fier Indien libre et respectueux de chacun), que sur des productions modernes grâce aux technologies de la communication. Dans ce contexte sont créées de véritables communautés web, autour des "Native" canadiens et américains, et sont inventés des média télévisuels avec le Aboriginal Network: une chaîne

40 Notamment à travers la reproduction mécanisée, la virtualité d'Internet où des oeuvres peuvent être éphémères, ou encore l'utilisation du numérique permettant retouches et transformations à volonté. 
diffusée aux Etats-Unis et au Canada, dont les contenus proviennent de toutes les régions et nations indiennes, de toutes les langues, qui sont ensuite traduites par le médium commun de l'anglais.

L'Indianité en tant qu'identité culturelle en genèse a besoin d'une langue commune, et c'est la langue du colon devenue leur qui fait office de lingua franca. On voit donc ici à l'oeuvre, dans la revendication de modalités culturelles empruntées puis réappropriées, l'idée d'une accession à une certaine idée de l'universel profondément liée aux particularités et non pas dans leur négation (l'humanisme critique de Todorov que j'évoquais plus haut). Le concept d'ethnoscape permet d'envisager la perspective dynamique de cette génèse perpétuelle, où les identités et même l'Indianité sont en constante réélaboration. Dans l'Indianité, la localité n'est donc plus celle d'un territoire tribal ou national, mais bien une localité imaginée : celle du continent nordaméricain et même du monde tout entier.

Marie GOYON

\section{BIBLIOGRAPHIE}

$\underline{\text { Retour à la table des matières }}$

APPADURAÏ Arjun, Après le colonialisme. Les conséquences culturelles de la globalisation, Payot, Paris, 2001.

ARDENNE Paul, Art, l'âge contemporain. Une histoire des arts plastiques à la fin du XXè siècle. Ed du Regard, Paris, 2003.

BATTISTE Marie et YOUNGBLOOD James, Protecting Indigenous Knowledge and Heritage. A global Challenge, Purich Publishing, Saskatoon, 2000. 
BOAS Franz, L’art primitif, Adam Biro, Paris, 2003.

BONTE P. et IZARD M. (ed), Dictionnaire de l'ethnologie et de l'anthropologie, PUF, Paris, 2000.

CLIFFORD James, Malaise dans la culture. L'ethnographie, la littérature et l'art au XXème siècle, ENS Beaux-Arts, Paris, 1996.

DELORIA Philippe J., Playing Indian, Yale University Publishing, Yale, 1998.

DESVEAUX Emmanuel, “Un guide sur la question de l'autre”, in "Lévi-Strauss, l'ethnologie ou la passion des autres", Magazine Littéraire, Hors série $n^{\circ} 4$, 2003, pp : 48-51.

DESVEAUX Emmanuel, « Les Grands Lacs et les Plaines, le figuratif et le géométrique, les hommes et les femmes », Papers of the 24th Algonquian Conference, 1993, 104-111.

DUPUIS Renée, La question indienne au Canada, Editions Boréal, Montréal, 1991.

DURHAM Jimmie, A certain Lack of Coherence : Writings on Art and Cultural Politics, Kala Press, Londres, 1993.

EDELMAN Murray, From Art to Politics : How artistic Creations shape political Conceptions, University of Chicago Press, Chicago, 1995.

FRANCIS Daniel, The imaginary Indian. The Image of the Indian in canadian Culture, Arsenal Pulp Press, Vancouver, 1992. 
GOYON Marie, «La logique du lien dans l'art du travail aux piquants des Amérindiens des Prairies ", Recherches Amérindiennes au Québec, volume XXXVI, n¹, 2006.

GRANT Agnes, No End of Grief. Indian Residential Schools in Canada, Pemmican Books, Winnipeg, 1996.

HERTZBERG Hazel W., The Search of an American Indian Identity. Modern Pan-Indian Movements, Syracuse University Press, Syracuse, 1972.

MacCLANCY Jeremy (ed), Contesting Art. Politics and Identity in the modern World, Berg, Oxford, 1997.

MacMASTER Gerald (ed), Indigena. Contemporary Native Perspectives in canadian Art, Craftsman House, New-York, 1992.

MARIENSTRAS Elise, La résistance indienne aux Etat-Unis du XVIème au Xxe siècle, Gallimard, Paris, 1980. 23

MEN (collectif), Le musée cannibale, GHK Editeurs, Neûchatel, 2002. NAGEL Joane, American Indian Ethnic Renewal. Red Power and the Resurgence of Identity and Culture, Oxford University Press, Oxford, 1996.

PETTIPAS Katherine, Severing the Ties that Bind. Government Repression of Indigenous Religious Ceremonies on the Prairies, The University of Manitoba Press, Winnipeg, 1994.

ROSTKOWSKI Joëlle, Le renouveau indien aux Etats-Unis, L'Harmattan, Paris, 1986.

ROSTKOWSKI Joëlle, L'indianité, contextes et perspectives, Presses Universitaires de Nancy, 1988. 
SILVER Shirley et MILLER Wick, American Indian Languages. Cultural and Social Contexts, University of Arizona Press, Tucson, 1997.

TODOROV Tzvetan, Nous et les autres. La réflexion française sur la diversité culturelle., Ed du Seuil, Paris, 1989.

TRIGGER Bruce, Les Indiens, la fourrure et les Blancs. Français et Amérindiens en Amérique du Nord, Boréal Seuil, Paris, 1992.

UZEL J.P, «L'art contemporain autochtone, point aveugle de la modernité » in BELLAVANCE Guy (ed), Monde et réseaux de l'art. Diffusion, migration et cosmopolitisme en art contemporain, Liber, Montréal, 2000, pp :189-203.

WADE Edwin L. (ed), The Arts of the North American Indian, Native Traditions in Evolution, Hudson Hills Press, New-York, 1985.

\section{Fin du texte}

